Check for updates

Cite this: RSC Adv., 2017, 7, 28499

Received 2nd April 2017

Accepted 20th May 2017

DOI: $10.1039 / c 7 r a 03795 c$

rsc.li/rsc-advances

\section{The mechanical and thermodynamic properties of $\beta-\mathrm{Si}_{1-x} \mathrm{C}$}

\author{
Qiang Zhao, (D) *a Zheng Zhang, ${ }^{a}$ Yang $\mathrm{Li}^{\mathrm{a}}$ and Xiaoping Ouyang ${ }^{\mathrm{abc}}$
}

By using the first-principles calculation method based on density functional theory (DFT), we investigated the stability, mechanical properties and thermodynamic performance of the carbon-rich $\beta-\mathrm{Si}_{1-x} \mathrm{C}$. Our results show that the volume of the $\beta-\mathrm{Si}_{1-x} \mathrm{C}$ crystal decreases when the $x$ value increases, while the density of $\beta-\mathrm{Si}_{1-x} \mathrm{C}$ increases when the $x$ value increases. When the $x$ value is smaller than 0.8148 , the formation energy of $\beta-\mathrm{Si}_{1-x} \mathrm{C}$ increases when the $x$ value increases, whereas when the $x$ value is larger than 0.8148 , the formation energy decreases when the $x$ value increases. The binding energy value of $\beta$ $\mathrm{Si}_{1-x} \mathrm{C}$ declines with the increase of $x$ value, which indicates that the stability of $\beta-\mathrm{Si}_{1-x} \mathrm{C}$ decreased as the $x$ value increases. The bulk modulus, shear modulus and Young modulus increase upon increasing the $x$ value, but the Poisson's ratio of $\beta-\mathrm{Si}_{1-x} \mathrm{C}$ decreases when the $x$ value increases. There is a nearly linear relationship between the thermodynamic properties of $\beta-\mathrm{Si}_{1-x} \mathrm{C}$ and the $x$ value, and the change in the thermodynamic properties is mainly due to the change in the lattice vibration. Our results provide theoretical support for the development of $\beta$-SiC.

\section{Introduction}

Silicon carbide (SiC) has received more and more attention since the 1890 s when it was discovered. SiC is a promising material that is used in the nuclear industry due to its excellent properties such as high corrosion resistance, good thermal conductivity, low neutron absorption cross section and retention rate of the fission gas. SiC is not only a cladding material ${ }^{1-5}$ that is used in light water reactors or a nuclear fuel coating material ${ }^{6-14}$ that is used in high temperature gas cooled reactors, but is also used in the structure of first wall materials ${ }^{15-21}$ that are used in fusion devices. SiC has many polytypes depending on the different stacking of the Si-C close-packed atomic plane..$^{22-24}$ More than 200 polytypes have currently been reported from the wide variety of preferred stacking sequences. ${ }^{25}$ The most common polytypes are $3 \mathrm{C}, 4 \mathrm{H}, 6 \mathrm{H}$ and $15 \mathrm{R}$, where the leading number shows the repetition of the $\mathrm{Si}-\mathrm{C}$ pair and where $\mathrm{C}, \mathrm{H}$ and $\mathrm{R}$ represent cubic, hexagonal and rhombohedral crystals, respectively. The $3 \mathrm{C}-\mathrm{SiC}$ crystal is known as $\beta$-SiC, and our research in this paper is based on the 3C polytype matrix.

With the development of the nuclear industry, research into $\mathrm{SiC}$ has never stopped. Y. Katoh, ${ }^{17}$ R. H. Jones ${ }^{26}$ and A. Hasegawa $^{27}$ studied the application of $\mathrm{SiC}$ ceramic materials in the

${ }^{a}$ Beijing Key Laboratory of Passive Safety Technology for Nuclear Energy, North China Electric Power University, Beijing 102206, P. R. China. E-mail: qzhao@ncepu.edu.cn; Fax: +8610 61773156; Tel: +861061773165

${ }^{b}$ Northwest Institute of Nuclear Technology, Xi'an 710024, P. R. China

${ }^{c}$ School of Materials Science and Engineering, Xiangtan University, Xiangtan 411105, P. R. China fusion field, and they found the critical issue of the application of SiC in the fusion field. L. L. Snead, ${ }^{28}$ G. Newsome ${ }^{29}$ and Y. $\mathrm{Katoh}^{30}$ studied the influence of neutron irradiation on SiC; their results showed that the swelling of SiC is associated with the dose of neutrons and the experimental temperature, and that the strength of SiC decreases after neutron irradiation. K. A. Terrani $^{31}$ and J. G. Stone ${ }^{3}$ studied SiC that was used as fuel cladding. Their results indicated that the dissolved oxygen activity in water can greatly accelerate the performance degradation of $\mathrm{SiC}$ since it has a large reaction rate constant. Although irradiation of the particles led to swelling of the SiC, SiC provides a reliable, impermeable barrier to the release of fission products. All of the research works demonstrate that the application of silicon carbide in the nuclear industry is reasonable. There are a lot research works focusing on SiC that use the molecular dynamics method. ${ }^{32-35}$ However, a firstprinciples study on $\mathrm{SiC}$ requires more effort. Recent studies have shown that carbon fiber can reinforce $\mathrm{SiC}$ and improve its performance, and some scientists have studied SiC with different $\mathrm{Si} / \mathrm{C}$ ratios using the molecular dynamics method. ${ }^{36,37}$ In this paper, we investigate the stability, mechanical and thermodynamic properties of $\beta-\mathrm{Si}_{1-x} \mathrm{C}$ by using the firstprinciples calculation method.

\section{Computational details}

Using the first-principles calculation method based on density functional theory (DFT), ${ }^{38,39}$ as implemented in the Cambridge serial total energy package (CASTEP) ${ }^{40}$ we investigated the stability, mechanical and thermodynamic properties of $\beta$ - 
$\mathrm{Si}_{1-x} \mathrm{C}$. CASTEP is an ab initio quantum mechanics theory software package based on DFT. The interaction between ions and electrons is described by the plane augmented wave (PAW) pseudopotential method, and the electron wave function is spread by plane wave basis sets. The exchange and correlation functions among the electrons are described by either a local density approximation (LDA) or a generalized gradient approximation (GGA). In this paper we used the $\mathrm{GGA}^{41-44}$ with a Perdew-Burke-Ernzerhol (PBE) ${ }^{45}$ form to describe the exchange and correlation functions among the electrons. After convergence tests, the parameters of our calculations were chosen as follows: the plane wave cutoff equals $500 \mathrm{eV}$, the space representation is reciprocal, the SCF tolerance equals $1.0 \times 10^{-6} \mathrm{eV}$ per atom, and $k$ sampling with a $3 \times 3 \times 3 k$-point mesh in the Brillouin zone was used. The optimal atomic positions were determined by satisfying the following conditions: (1) the maximum force on the atoms was smaller than $0.05 \mathrm{eV} \mathrm{nm}^{-1}$, (2) the maximum change of the energy per atom was smaller than $1.0 \times 10^{-5} \mathrm{eV},(3)$ the maximum displacement was smaller than $0.001 \AA$ and (4) the maximum stress of the crystal was smaller than $0.02 \mathrm{GPa}$. All of the properties of $\beta$-SiC that we calculated in this paper are based on the optimized crystal structure.

Phonon calculations were carried out using the linear response method (density functional perturbation theory) applied with a norm-conserving pseudo-potential. A $q$-point grid of $10 \times 10 \times 10$ was applied along with a cutoff energy of $500 \mathrm{eV}$ for $\beta-\mathrm{Si}_{1-x} \mathrm{C}$. The thermodynamic properties of $\beta-\mathrm{Si}_{1-x} \mathrm{C}$ were obtained via the resultant phonon dispersion curve. Based on the phonon calculations, the enthalpy $(H)$, free energy $(F)$, entropy $(S)$ and heat capacity $\left(C_{\mathrm{V}}\right)$ as functions of temperature at a finite temperature could be computed. The vibrational contributions to the thermodynamic parameters were evaluated using Debye's quasi-harmonic approximation:

$$
\begin{gathered}
H(T)=E_{\mathrm{tot}}-E_{\mathrm{ZP}}+\int \frac{h \omega}{\exp (h \omega / k T)} F(\omega) \mathrm{d}(\omega) \\
F(T)=E_{\mathrm{tot}}-E_{\mathrm{ZP}}+k T \int \ln [1-\exp (-h \omega / k T)] F(\omega) \mathrm{d}(\omega) \\
S(T)=k \int \frac{h \omega / k T}{\exp (h \omega / k T)} F(\omega) \mathrm{d}(\omega) \\
-k \int[1-\exp (-h \omega / k T)] F(\omega) \mathrm{d}(\omega) \\
C_{\mathrm{V}}(T)=k \int \frac{(h \omega / k T)^{2} \exp (h \omega / k T)}{\exp (h \omega / k T)-1} F(\omega) \mathrm{d}(\omega)
\end{gathered}
$$

where $E_{\text {tot }}$ is the total energy of the system, $E_{\mathrm{ZP}}$ is the zero point vibrational energy, $\omega$ is the vibration frequency of the phonon model in the Brillouin zone, $F(\omega)$ is the phonon density of states, and $k$ and $h$ are the Boltzmann constant and Planck constant, respectively.

The Debye temperature, $\Theta_{\mathrm{D}}$, can be estimated from analysis of the heat capacity using the following equations:

$$
\begin{gathered}
C_{\mathrm{V}}(T)=9 n k\left(\frac{T}{\Theta_{\mathrm{D}}}\right)^{3} \int_{0}^{\frac{\Theta_{\mathrm{D}}}{T}} \frac{x^{4} \mathrm{e}^{x}}{\left(\mathrm{e}^{x}-1\right)^{2}} \mathrm{~d} x \\
x=\frac{h \omega}{k T}
\end{gathered}
$$

where $n$ is the number of atoms in the cell.

$\beta$-SiC is usually known as $3 \mathrm{C}-\mathrm{SiC}$ and it possesses a cubic phase zinc blende structure. Every atom in SiC is surrounded by four heterogeneous atoms and they combine together through the directional strong tetrahedral $\mathrm{sp}^{3}$ bonds. The space group of $\beta$-SiC is F43m (196) and its crystal structure is shown in Fig. 1. In the cell, silicon atoms are located in the cubic vertex angles and the face centres and carbon atoms are located in the sites that are a quarter of the diagonal. In order to study the stability, mechanical and thermodynamic properties of $\beta-\mathrm{Si}_{1-x} \mathrm{C}$, we choose a $2 a_{0} \times 2 a_{0} \times 2 a_{0}$ supercell, where $a_{0}$ is the lattice constant of a perfect $\beta$-SiC cell. To study the stability, mechanical and thermodynamics properties of $\beta-\mathrm{Si}_{1-x} \mathrm{C}$ with a series of different $x$ values, we randomly replaced two silicon atoms with carbon atoms at every step, and we made sure to do this in a symmetrical manner as much as possible, e.g., the first silicon atom that will be replaced by a carbon atom is in the upper left corner of the supercell, the next silicon atom is in the lower right corner. Our calculations showed that the substituted carbon atoms preferred to be far away from each other in the supercell because the total energy of the supercell (when the substituted carbon atoms are far away from each other) was lower than that of the supercell with the substituted carbon atoms clustered together. When all of the silicon atoms in $\beta$-SiC were replaced by carbon atoms, we referred to it as diamond-C.

\section{Results and discussion}

\subsection{Geometry optimization results}

By optimizing the supercell, we can determine the stable structure of $\beta-\mathrm{Si}_{1-x} \mathrm{C}$. The lattice constants and the densities of $\beta$-Si ${ }_{1-x} \mathrm{C}$ are shown in Fig. 2 and Table 1. Table 1 shows that our

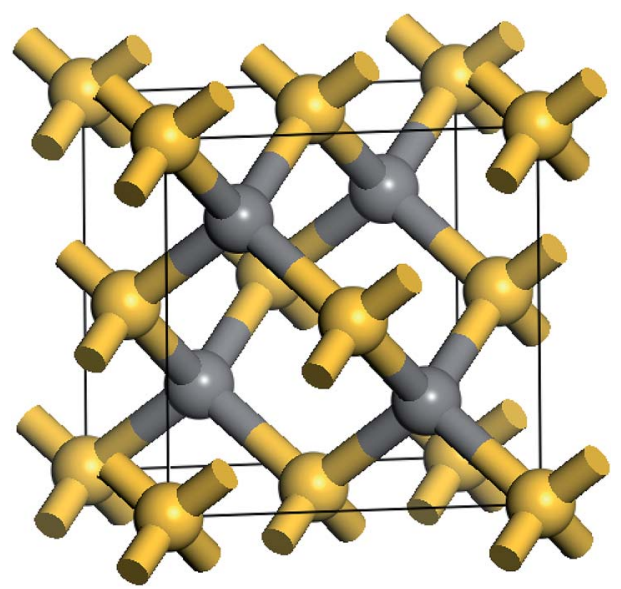

Fig. 1 Schematic diagram of the $\beta$-SiC cell structure; the golden and gray balls correspond to silicon and carbon atoms, respectively. 


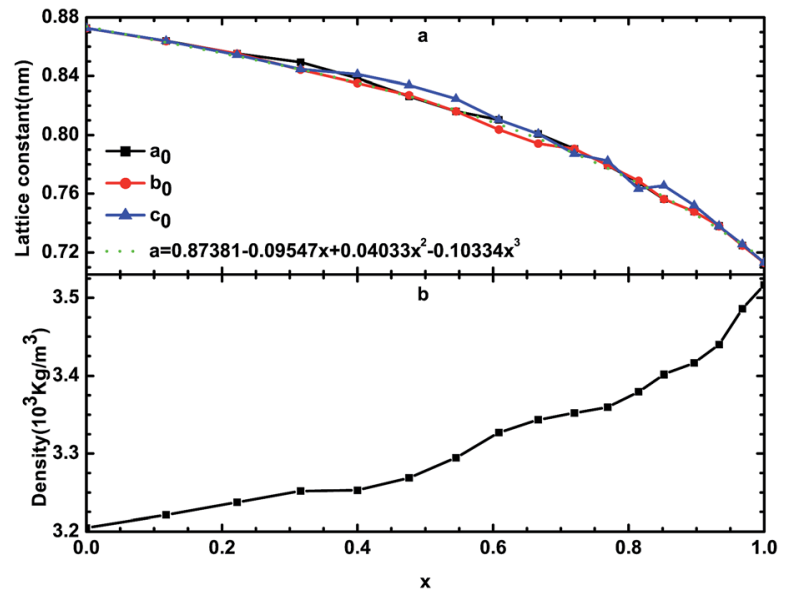

Fig. 2 Lattice constants (a) and densities (b) of $\beta-\mathrm{Si}_{1-x} \mathrm{C}_{;} \mathrm{a}_{0}, b_{0}$ and $\mathrm{c}_{0}$ are the lattice constants of $\beta-\mathrm{Si}_{1-x} \mathrm{C}$; the dotted line is the line of best fit.

calculated lattice constants are in good agreement with the results of other researchers. Fig. 2(a) shows that the lattice constants decrease when the $x$ value increases; this result is caused by the difference in the size of the carbon atoms and silicon atoms. We can describe the relationship between the lattice constants of $\beta-\mathrm{Si}_{1-x} \mathrm{C}$ and the $x$ value using the equation $a=0.87381-0.09547 x+0.04033 x^{2}-0.10334 x^{3}$. Fig. 2(b) shows that the density of $\beta-\mathrm{Si}_{1-x} \mathrm{C}$ increases when the $x$ value increases; this is because the decline rate of the mass of the $\beta$ $\mathrm{Si}_{1-x} \mathrm{C}$ supercell is less than that of the volume.

\subsection{Formation energy and binding energy}

The formation energy and binding energy are two important parameters that reflect the stability of the $\beta-\mathrm{Si}_{1-x} \mathrm{C}$ crystal structure. The computational formulae for calculating the crystal formation energy and binding energy are as follows:

$$
\begin{aligned}
& E_{\mathrm{f}}=\frac{1}{x+y}\left[E_{\mathrm{tot}}^{\mathrm{AB}}-\left(x E_{\mathrm{solid}}^{\mathrm{A}}+y E_{\mathrm{solid}}^{\mathrm{B}}\right)\right] \\
& E_{\mathrm{b}}=\frac{1}{x+y}\left[E_{\mathrm{tot}}^{\mathrm{AB}}-\left(x E_{\mathrm{atom}}^{\mathrm{A}}+y E_{\mathrm{atom}}^{\mathrm{B}}\right)\right]
\end{aligned}
$$

where $E_{\mathrm{f}}$ and $E_{\mathrm{b}}$ correspond to the formation energy and binding energy, respectively, of $\beta-\mathrm{Si}_{1-x} \mathrm{C}, E_{\mathrm{tot}}^{\mathrm{AB}}$ is the total energy of the crystal that is composed of the elements A (Si) and B (C), $x$ and $y$ are the total numbers of the elements $\mathrm{A}$ and $\mathrm{B}, E_{\text {solid }}^{\mathrm{A}}$ and $E_{\text {solid }}^{\mathrm{B}}$ are the atomic energies of A and B in a monocrystal, and $E_{\mathrm{atom}}^{\mathrm{A}}$ and $E_{\mathrm{atom}}^{\mathrm{B}}$ are the energies of the isolated atoms $\mathrm{A}$ and $\mathrm{B}$, respectively. On the one hand, the formation energy reflects the stability of a crystal structure. On the other hand, it also reflects how difficult it is to form the crystal structure. The smaller the formation energy, the more stable the crystal structure is and the easier it is to form the structure. The larger the value of the binding energy, the more stable the crystal structure is. To quantitatively analyse the stabilities of the $\beta$-Si $i_{1-x} \mathrm{C}$ crystals, we calculated their formation and binding energies and the results are shown in Fig. 3. The formation energy increased when the $x$ value increased, up to an $x$ value of 0.8148 . This result indicates that the formation of $\beta-\mathrm{Si}_{1-x} \mathrm{C}$ becomes more difficult when $x$ increases. When the $x$ value was larger than 0.8148 , the formation energy decreased when the $x$ value increased. The formation energy results demonstrate that the stability of $\beta$ $\mathrm{Si}_{1-x} \mathrm{C}$ decreases when $x$ is increased. The binding energy of $\beta$ $\mathrm{Si}_{1-x} \mathrm{C}$ decreases when the $x$ value is increased, which also shows that the stability of the crystal structure decreases when the $x$ value is increased.

\subsection{Mechanical properties}

As an important structural material that is used in the nuclear industry, the mechanical properties of $\beta$-SiC are very important.

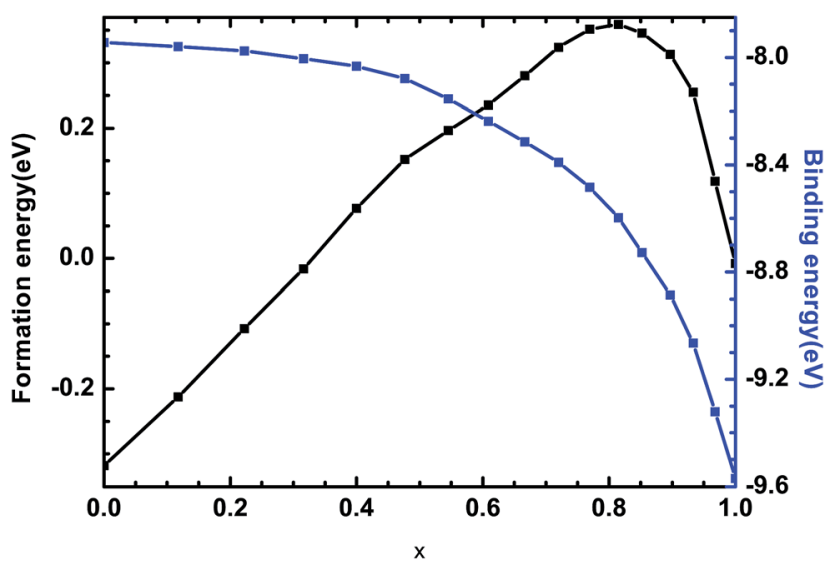

Fig. 3 Formation energies and binding energies of $\beta-\mathrm{Si}_{1-x} \mathrm{C}$.

Table 1 Lattice parameters and elastic modulus values of $\beta$-SiC and diamond-C, with our results compared with other theoretical and

\begin{tabular}{|c|c|c|c|c|c|}
\hline Symbol & $a_{0} / \mathrm{nm}$ & $B / \mathrm{GPa}$ & $G / \mathrm{GPa}$ & $E / \mathrm{GPa}$ & $\nu$ \\
\hline$\beta$-SiC & 0.436 & 212.519 & 189.643 & 324.026 & 0.246 \\
\hline $\mathrm{Cal}^{46}$ & 0.433 & 215 & 181.8 & 425.3 & 0.17 \\
\hline Exp. & 0.436 (ref. 48 ) & 225 (ref. 49) & 192 (ref. 50) & 314.2 (ref. 51) & 0.267 (ref. 49) \\
\hline Diamond-C & 0.357 & 431.206 & 523.598 & 1029.379 & 0.102 \\
\hline $\mathrm{Cal}^{52}$ & 0.358 & 406.776 & 542.571 & 1046.993 & 0.071 \\
\hline
\end{tabular}
experimental results 
We calculated the mechanical properties of $\beta-\mathrm{Si}_{1-x} \mathrm{C}$ with a series of different $x$ values. Table 1 shows the mechanical properties of $\beta$-SiC and diamond-C; the results of our calculations are in good agreement with the results from other theoretical calculations and experimental data. Fig. 4 shows the mechanical properties of $\beta-\mathrm{Si}_{1-x} \mathrm{C}$ with different $x$ values. On the whole, the values of the bulk modulus, shear modulus and Young's modulus of $\beta-\mathrm{Si}_{1-x} \mathrm{C}$ increase when the $x$ value is increased, and the Poisson's ratio of $\beta-\mathrm{Si}_{1-x} \mathrm{C}$ decreases when the $x$ value is increased. To our knowledge, the material is ductile if the value of $G / B$ is smaller than 0.57 , and the ductility of a material increases when the value of $G / B$ decreases. The material is brittle if the value of $G / B$ is larger than 0.57 ; the larger the value, the greater the brittleness of the material. The results of our calculations show that the value of $G / B$ changes from 0.885 to 1.204 upon increasing the $x$ value. No matter how large the value of $x$ is, the $\beta-\mathrm{Si}_{1-x} \mathrm{C}$ crystal is a brittle material. $\beta$ $\mathrm{Si}_{1-x} \mathrm{C}$ becomes more and more brittle when the $x$ value is increased, and we come to the same conclusion from the change in the Poisson's ratio. In Fig. 4(c), there are some values of the Young's modulus and the Poisson's ratio that are different from the general trends. The reason for this phenomenon is the asymmetry of the silicon atoms' positions as the silicon atoms are replaced by carbon atoms. In order to discuss the effects of supercell size on the results, we used $1 a \times$ $1 a \times 1 a$ (8-atoms), $2 a \times 2 a \times 2 a$ (64-atoms) and $3 a \times 3 a \times 3 a$ (216-atoms) supercell models to check our conclusions in this paper. Fig. 5 shows the mechanical properties of $\beta-\mathrm{Si}_{1-x} \mathrm{C}$ based on a series of different supercell sizes, and the results show that the supercell size does not affect the conclusions about the mechanical properties of $\beta-\mathrm{Si}_{1-x} \mathrm{C}$. The general trends shown in Fig. 4 and 5, of the elastic modulus values upon increasing the $x$ value, can be explained by the differences in the bond strengths. The strengths of the bonds between carbon atoms and silicon atoms follow this rule: $\mathrm{C}-\mathrm{C}>\mathrm{C}-\mathrm{Si}>\mathrm{Si}-\mathrm{Si}$. The higher the number of silicon atoms that are replaced by carbon atoms, the more $\mathrm{C}-\mathrm{C}$ bonds there are in the $\beta-\mathrm{Si}_{1-x} \mathrm{C}$ crystal. Therefore, the $\beta$-Si ${ }_{1-x} \mathrm{C}$ crystal becomes more difficult to destroy when the $x$

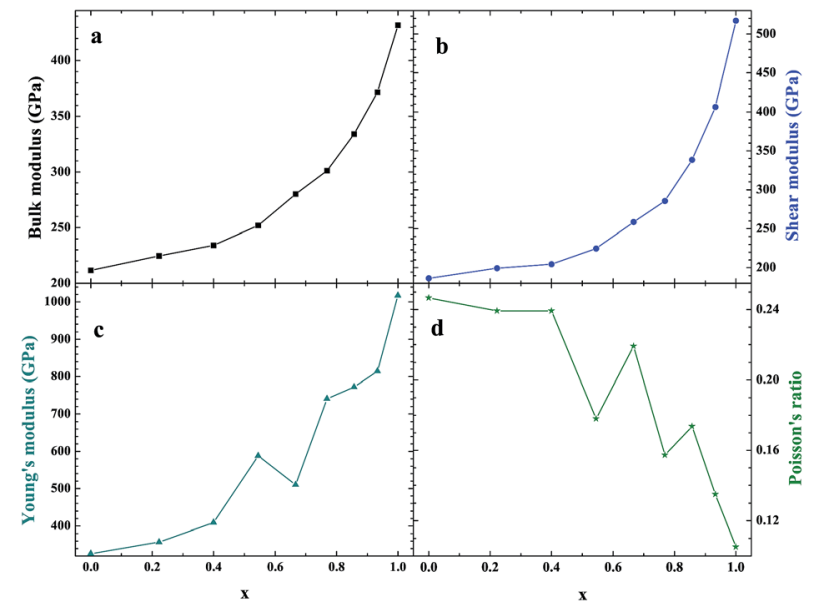

Fig. 4 Mechanical properties of $\beta-\mathrm{Si}_{1-x} \mathrm{C}$; bulk modulus (a), shear modulus (b), Young's modulus (c) and Poisson's ratio (d).

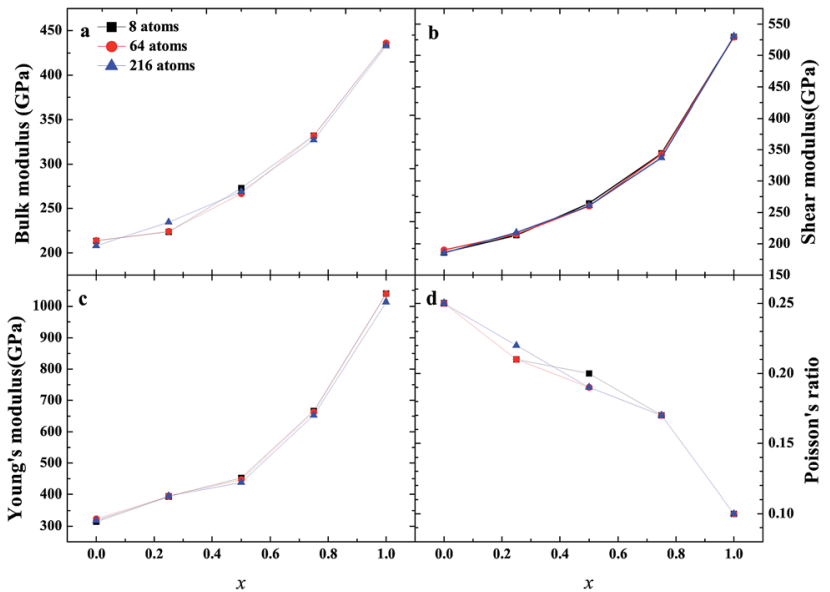

Fig. 5 Mechanical properties of $\beta-\mathrm{Si}_{1-x} \mathrm{C}$ based on different supercell sizes; bulk modulus (a), shear modulus (b), Young's modulus (c) and Poisson's ratio $(d)$.

value increases, and the hardness of $\beta-\mathrm{Si}_{1-x} \mathrm{C}$ increases when the $x$ value increases.

\subsection{Thermodynamic properties}

No matter where $\beta$-SiC as a structural material is used, whether in a fission reactor or in a fusion device, the thermodynamic properties of $\beta$-SiC are very important for its applications. We calculated the thermodynamic properties of $\beta-\mathrm{Si}_{1-x} \mathrm{C}$ in order to investigate the effects of carbon content on the thermodynamic properties of $\beta$-SiC. Fig. $6(\mathrm{a})$ and (b) show the phonon dispersion curves of $\beta$-SiC and diamond-C, respectively. The results of the phonon dispersion curve of $\beta$-SiC are in good agreement with Karch's computational results. ${ }^{55-58}$ The difference between the phonon dispersion curves of $\beta$-SiC and diamond-C is not obvious, however the frequency of diamond-C is broader when compared with that of $\beta$-SiC. The phonon dispersion curves of all of the $\beta-\mathrm{Si}_{1-x} \mathrm{C}$ examples were calculated, however we only show the phonon dispersion curves of $\beta$-SiC and diamond-C in Fig. 6. Our results show that there is no imaginary frequency,

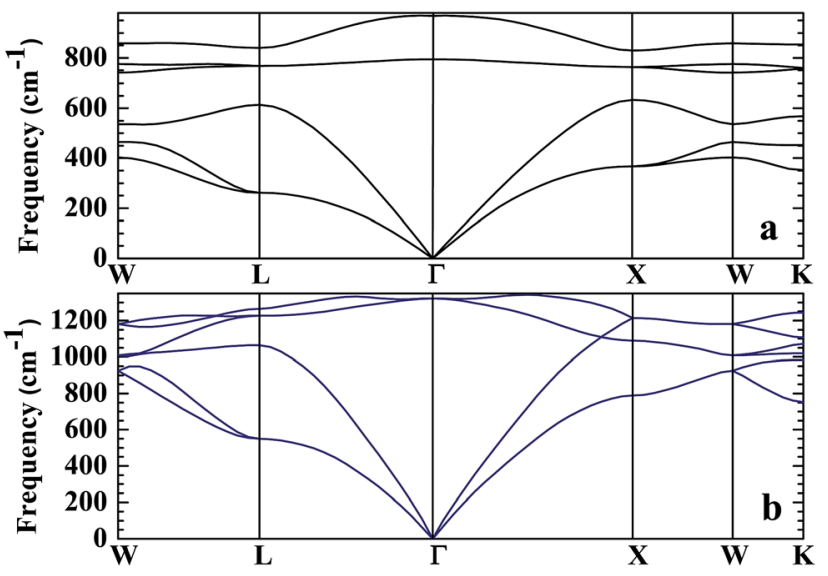

Fig. 6 Phonon dispersion curves of $\beta-\operatorname{SiC}(a)$ and diamond-C (b). 
which indicates that the structures of all of the $\beta-\mathrm{Si}_{1-x} \mathrm{C}$ examples are stable. The Debye temperature is an important physical property for materials, as it not only represents the interactions of atoms in materials, but is also closely related to the Young's modulus, dilatation coefficients, specific heat and thermal conductivity. Fig. 7 shows the Debye temperatures of $\beta-\mathrm{Si}_{1-x} \mathrm{C}$, and the results show that the Debye temperature of $\beta-\mathrm{Si}_{1-x} \mathrm{C}$ increases when the $x$ value increases, which is in good agreement with the change in the Young's modulus, which also increases when the $x$ value increases. The Debye temperature of $\beta$-SiC determined from our calculations is in good agreement with the reported value in ref. 59.

Based on phonon calculations and the Debye quasiharmonic approximation, we calculated the enthalpy, free energy, entropy and heat capacity of $\beta$-Si ${ }_{1-x} \mathrm{C}$. Fig. 8 shows the enthalpy, free energy, entropy and heat capacity of $\beta-\mathrm{Si}_{1-x} \mathrm{C}$, respectively. At the same temperature the value of the free energy increases when the $x$ value increases, and the enthalpy increases almost linearly with temperature and tends to display $K_{\mathrm{b}} T$ behavior. The entropy increases slowly with increasing temperature when the temperature is below $300 \mathrm{~K}$, and it increases rapidly when the temperature is above $300 \mathrm{~K}$. As a whole, the enthalpy, entropy and heat capacity of $\beta-\mathrm{Si}_{1-x} \mathrm{C}$ decreases when the $x$ value increases. The enthalpy of $\beta-\mathrm{Si}_{1-x} \mathrm{C}$ decreases when the $x$ value increases, which suggests that the total energy of $\beta-\mathrm{Si}_{1-x} \mathrm{C}$ decreases when the $x$ value increases. The free energy corresponds to the work outside that is converted from the system internal energy in the thermodynamics process. The value of the free energy of $\beta-\mathrm{Si}_{1-x} \mathrm{C}$ increases when the $x$ value increases, which means that reducing the internal energy converts less work outside when the $x$ value increases. The change of the free energy also demonstrates that the stability of $\beta-\mathrm{Si}_{1-x} \mathrm{C}$ becomes poorer and poorer, especially in high temperature region. The entropy represents the system energy that is not available. Fig. 8(c) shows that the entropy decreases when the $x$ value increases, which demonstrates that less energy is not available when $x$ increases. The heat capacity is the absorption energy when the system rises by one degree

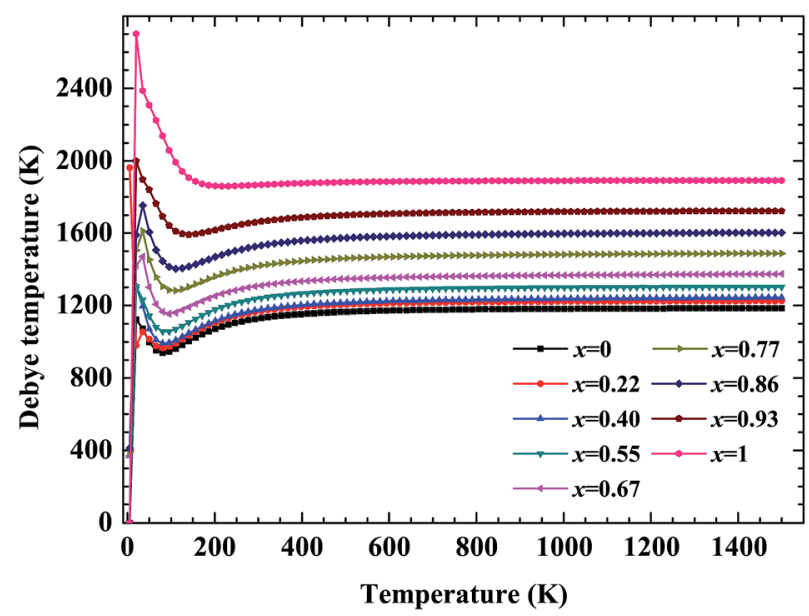

Fig. 7 Debye temperatures of $\beta-\mathrm{Si}_{1-x} \mathrm{C}$.

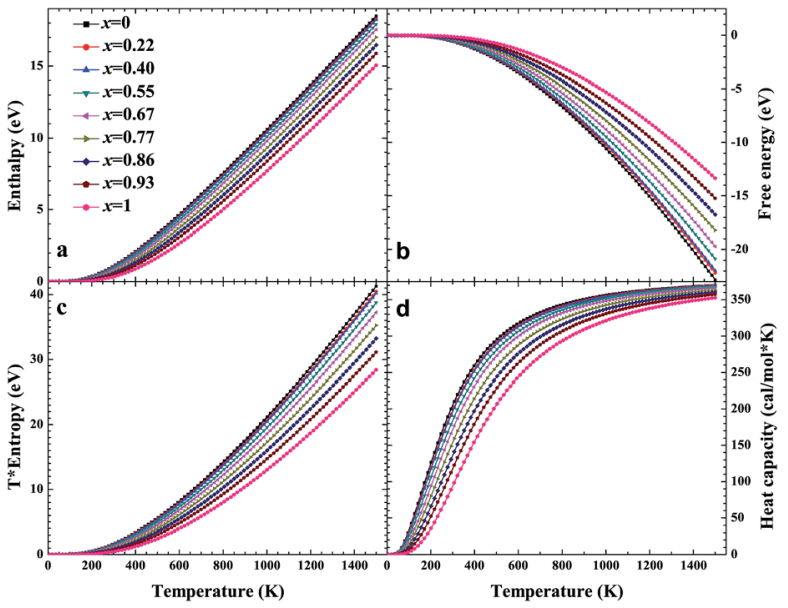

Fig. 8 Enthalpy (a), free energy (b), entropy (c) and heat capacity (d) of $\beta-\mathrm{Si}_{1-x} \mathrm{C}$.

centigrade. The heat capacity of $\beta-\mathrm{Si}_{1-x} \mathrm{C}$ decreases when $x$ increases; this result means that it becomes easier to heat $\beta$ $\mathrm{Si}_{1-x} \mathrm{C}$ when the $x$ value increases. The thermal conductivity of $\mathrm{SiC}$ arises mainly through lattice vibration, and we can deduce the heat conductivity of $\beta$-SiC according to the phonon heat capacity. The reasons for the change in the thermodynamic properties are that the number of silicon atoms and carbon atoms changes when $x$ increases, and the difference in atoms leads to the diversity of phonon frequency in the Brillouin zone for the $\beta-\mathrm{Si}_{1-x} \mathrm{C}$ structure.

\section{Conclusion}

In this paper we studied the stability, mechanical and thermodynamic properties of $\beta-\mathrm{Si}_{1-x} \mathrm{C}$ via the first-principles calculation method, and the results were as follows:

(1) The supercell volume of $\beta$-Si ${ }_{1-x} \mathrm{C}$ decreases when the $x$ value increases, but the density of $\beta-\mathrm{Si}_{1-x} \mathrm{C}$ increases when the $x$ value increases.

(2) The stability of $\beta-\mathrm{Si}_{1-x} \mathrm{C}$ becomes poorer and poorer when the $x$ value increases.

(3) The bulk modulus, shear modulus and Young's modulus of $\beta$-Si ${ }_{1-x} \mathrm{C}$ increases when the $x$ value increases, and the Poisson's ratio decreases when $x$ increases. $\beta-\mathrm{Si}_{1-x} \mathrm{C}$ becomes more brittle when the $x$ value increases.

(4) The Debye temperature and free energy of $\beta-\mathrm{Si}_{1-x} \mathrm{C}$ increases when the $x$ value increases, and the enthalpy, entropy and heat capacity of $\beta-\mathrm{Si}_{1-x} \mathrm{C}$ decreases as the $x$ value increases.

All of our results are in good agreement with the existing theoretical and experimental works. The change in the volume and density of $\beta-\mathrm{Si}_{1-x} \mathrm{C}$ is associated with the difference of the atomic size. All of the $\beta-\mathrm{Si}_{1-x} \mathrm{C}$ examples are stable from the point view of the binding energy. The change in the mechanical properties of $\beta-\mathrm{Si}_{1-x} \mathrm{C}$ are mainly due to the changing internal bonds in the supercell. The change in the thermodynamic properties is associated with the difference in the lattice vibration, and the difference arises from the different number of silicon and carbon atoms. Our results suggest that there is 
a linear relationship between the mechanical properties and the carbon content, and the thermodynamic properties and the carbon content also demonstrate a linear relationship. Our works might provide a theoretical support for the development of carbon fiber reinforced $\beta$-SiC.

\section{Acknowledgements}

This work was supported by the National Natural Science Foundation of China under Grant No. 11275071 and 11305061, and the Fundamental Research Funds for the Central Universities under Grant No. 2017MS079.

\section{References}

$1 \mathrm{H}$. Feinroth, A Multi-Layered Ceramic Composite for Impermeable Fuel Cladding for Commercial Water Reactors, Gamma engineering corporation technical report, 2008.

2 K. Yueh, D. Carpenter and H. Feinroth, Nucl. Eng. Int., 2010, 55, 14-16.

3 J. G. Stone, R. Schleicher, C. P. Deck, G. M. Jacobsen, H. E. Khalifa and C. A. Back, J. Nucl. Mater., 2015, 466, 682-697.

4 D. M. Carpenter, Ph.D thesis, Massachusetts Institute of Technology, 2010.

5 Y. Katoh, L. L. Snead, C. H. Henager, T. Nozawa, T. Hinoki, A. Iveković, S. Novak and S. G. de Vicente, J. Nucl. Mater., 2014, 455, 387-397.

6 R. Blackstone and E. H. Voice, J. Nucl. Mater., 1971, 39, 319322.

7 R. J. Price, J. Nucl. Mater., 1969, 33, 17-22.

8 R. J. Price, Nucl. Technol., 1977, 35, 320-336.

9 R. J. Price, J. Nucl. Mater., 1973, 48, 47-57.

10 W. Primak, L. H. Fuchs and P. P. Day, Phys. Rev., 1956, 103, 1184.

11 M. Balarin, Phys. Status Solidi B, 1965, 11, K67-K71.

12 D. Littler, Properties of reactor materials and the effects of radiation damage, Butterworths, 1962.

13 R. Stevens, Philos. Mag., 1972, 25, 523-528.

14 R. P. Thorne and V. C. Howard, Radiation-induced changes in porous cubic silicon carbide, Eng. Technical Report, United kingdom atomic energy authority, Culcheth, 1967.

15 C. H. Henager, B. N. Nguyen, R. J. Kurtz, T. J. Roosendaal, B. A. Borlaug, M. Ferraris, A. Ventrella and Y. Katoh, J. Nucl. Mater., 2015, 466, 253-268.

16 C. H. Henager and R. J. Kurtz, J. Nucl. Mater., 2011, 417, 375378.

17 Y. Katoh, L. L. Snead, C. H. Henager, A. Hasegawa, A. Kohyama, B. Riccardi and H. Hegeman, J. Nucl. Mater., 2007, 367, 659-671.

18 P. Colombo, B. Riccardi, A. Donato and G. Scarinci, J. Nucl. Mater., 2000, 278, 127-135.

19 S. Sharafat, R. H. Jones, A. Kohyama and P. Fenici, Fusion Eng. Des., 1995, 29, 411-420.

20 R. H. Jones and C. H. Henager, J. Nucl. Mater., 1995, 219, 5562.
21 M. Ferraris, M. Salvo, C. Isola, M. A. Montorsi and A. Kohyama, J. Nucl. Mater., 1998, 258, 1546-1550.

22 N. W. Jepps and T. F. Page, Prog. Cryst. Growth Charact., 1983, 7, 259-307.

23 P. Pirouz and J. W. Yang, Ultramicroscopy, 1993, 51, 189-214.

24 P. Shaffer, Acta Crystallogr., Sect. B: Struct. Crystallogr. Cryst. Chem., 1969, 25, 477-488.

25 G. R. Fisher and P. Barnes, Philos. Mag., 1990, 61, 217-236.

26 R. H. Jones, L. Giancarli, A. Hasegawa, Y. Katoh, A. Kohyama, B. Riccardi, L. L. Snead and W. J. Weber, J. Nucl. Mater., 2002, 307, 1057-1072.

27 A. Hasegawa, A. Kohyama, R. Jones, L. Snead, B. Riccardi and P. Fenici, J. Nucl. Mater., 2000, 283, 128-137.

28 L. L. Snead, Y. Katoh and S. Connery, J. Nucl. Mater., 2007, 367, 677-684.

29 G. Newsome, L. L. Snead, T. Hinoki, Y. Katoh and D. Peters, J. Nucl. Mater., 2007, 371, 76-89.

30 Y. Katoh, T. Nozawa, L. L. Snead, K. Ozawa and H. Tanigawa, J. Nucl. Mater., 2011, 417, 400-405.

31 K. A. Terrani, Y. Yang, Y.-J. Kim, R. Rebak, H. Meyer and T. J. Gerczak, J. Nucl. Mater., 2015, 465, 488-498.

32 H. Kikuchi, R. K. Kalia, A. Nakano, P. Vashishta, P. S. Branicio and F. Shimojo, J. Appl. Phys., 2005, 98, 103524.

33 F. Shimojo, I. Ebbsjö, R. K. Kalia, A. Nakano, J. P. Rino and P. Vashishta, Phys. Rev. Lett., 2000, 84, 3338.

34 F. Gao, H. Y. Xiao, X. T. Zu, M. Posselt and W. J. Weber, Phys. Rev. Lett., 2009, 103, 027405.

35 W. H. Lee, X. H. Yao, W. R. Jian and Q. Han, Comput. Mater. Sci., 2015, 98, 297-303.

36 A. Adnan and S. F. Ferdous, Comput. Mater. Sci., 2015, 110, 331-339.

37 A. Adnan and S. F. Ferdous, Comput. Mater. Sci., 2015, 96, 354-359.

38 P. Hohenberg and W. Kohn, Phys. Rev., 1964, 136, B864B871.

39 W. Kohn and L. J. Sham, Phys. Rev., 1965, 140, A1133-A1138.

40 S. J. Clark, M. D. Segall, C. J. Pickard, P. J. Hasnip, M. J. Probert, K. Refson and M. Payne, Z. Kristallogr., 2005, 220, 567-570.

41 J. P. Perdew and W. Yue, Phys. Rev. B: Condens. Matter Mater. Phys., 1986, 33, 8800.

42 J. P. Perdew, J. Chevary, S. Vosko, K. A. Jackson, M. R. Pederson, D. Singh and C. Fiolhais, Phys. Rev. B: Condens. Matter Mater. Phys., 1992, 46, 6671.

43 J. P. Perdew, K. Burke and Y. Wang, Phys. Rev. B: Condens. Matter Mater. Phys., 1996, 54, 16533.

44 H. Iikura, T. Tsuneda, T. Yanai and K. Hirao, J. Chem. Phys., 2001, 115, 3540-3544.

45 J. P. Perdew, K. Burke and M. Ernzerhof, Phys. Rev. Lett., 1996, 77, 3865.

46 E. Konstantinova, M. J. V. Bell and V. Anjos, Intermetallics, 2008, 16, 1040-1042.

47 X. J. Liu, L. F. Li and F. Lu, Physics, 2013, 1745, 1301-1313. $48 \mathrm{http}: / /$ www.ioffe.ru/SVA/NSM/Semicond/.

49 D. W. Feldman, J. H. Parker Jr, W. J. Choyke and L. Patrick, Phys. Rev., 1968, 173, 787.

50 R. D. Carnahan, J. Am. Ceram. Soc., 1968, 51, 223-224. 
51 P. Vashishta, R. K. Kalia, A. Nakano and J. P. Rino, J. Appl. Phys., 2007, 101, 103515.

52 X. J. Liu, L. F. Li, Q. Q. Li, Y. Li and F. Lu, Mater. Sci. Semicond. Process., 2013, 16, 1369-1376.

53 H. Yao, L. Ouyang and W.-Y. Ching, J. Am. Ceram. Soc., 2007, 90, 3194-3204.

54 H. J. McSkimin and P. Andreatch Jr, J. Appl. Phys., 1972, 43, 2944-2948.

55 K. Karch, P. Pavone, W. Windl, D. Strauch and F. Bechstedt, Int. J. Quantum Chem., 1995, 56, 801-817.
56 K. Karch, P. Pavone, W. Windl, O. Schütt and D. Strauch, Phys. Rev. B: Condens. Matter Mater. Phys., 1994, 50, 17054.

57 O. Madelung, U. RÃüssler and M. Schulz, Diamond (C) phonon dispersion relations, phonon density of states, Springer, Berlin Heidelberg, 2001, pp. 1-11.

58 B. Monserrat and R. J. Needs, Phys. Rev. B: Condens. Matter Mater. Phys., 2014, 89, 214304.

59 A. Zywietz, K. Karch and F. Bechstedt, Phys. Rev. B: Condens. Matter Mater. Phys., 1996, 54, 1791. 